\title{
Cooperative Astrocyte and Dendritic Spine Dynamics at Hippocampal Excitatory Synapses
}

\author{
Michael Haber, Lei Zhou, and Keith K. Murai \\ Centre for Research in Neuroscience, Department of Neurology and Neurosurgery, The Research Institute of the McGill University Health Centre, Montreal \\ General Hospital, Montreal, Quebec, H3G 1A4, Canada
}

Accumulating evidence is redefining the importance of neuron-glial interactions at synapses in the CNS. Astrocytes form "tripartite" complexes with presynaptic and postsynaptic structures and regulate synaptic transmission and plasticity. Despite our understanding of the importance of neuron-glial relationships in physiological contexts, little is known about the structural interplay between astrocytes and synapses. In the past, this has been difficult to explore because studies have been hampered by the lack of a system that preserves complex neuron-glial relationships observed in the brain. Here we present a system that can be used to characterize the intricate relationship between astrocytic processes and synaptic structures in situ using organotypic hippocampal slices, a preparation that retains the three-dimensional architecture of astrocyte-synapse interactions. Using time-lapse confocal imaging, we demonstrate that astrocytes can rapidly extend and retract fine processes to engage and disengage from motile postsynaptic dendritic spines. Surprisingly, astrocytic motility is, on average, higher than its dendritic spine counterparts and likely relies on actin-based cytoskeletal reorganization. Changes in astrocytic processes are typically coordinated with changes in spines, and astrocyte-spine interactions are stabilized at larger spines. Our results suggest that dynamic structural changes in astrocytes help control the degree of neuron-glial communication at hippocampal synapses.

Key words: plasticity; glia; motility; cross talk; neuron-glia; organotypic slices

\section{Introduction}

Structural modifications to cellular components are critical for establishing and remodeling connections within the nervous system. Recent studies have offered unprecedented views of structural changes at neuromuscular (Walsh and Lichtman, 2003; Bishop et al., 2004) and central (Dailey and Smith, 1996; Fischer et al., 1998; Dunaevsky et al., 1999; Niell et al., 2004) synapses and have instigated numerous investigations into the structural plasticity of neural connectivity. Interestingly, synapses in the CNS undergo morphological rearrangements even into adulthood (Grutzendler et al., 2002; Trachtenberg et al., 2002; Holtmaat et al., 2005; Zuo et al., 2005). In particular, dendritic spines, the small protrusions on dendrites that receive glutamatergic innervation, can change their geometry within minutes, providing rapid adjustments to postsynaptic ionic balance (Korkotian and Segal, 1998; Volfovsky et al., 1999), protein trafficking (Richards et al., 2004), and signaling events that regulate synaptic efficacy (Segal, 2005). Indeed, enlargements and reductions in spine size

Received March 27, 2006; revised June 21, 2006; accepted July 24, 2006.

This work was supported by the Canadian Institutes of Health Research, Canada Research Chairs Program, Canadian Foundation for Innovation, and the EJLB Foundation (K.K.M.). M.H. was supported by a studentship from the Research Institute of the McGill University Health Centre and the McGill Department of Medicine. We are grateful to R. Dunn, Q. Deng, K. Lundstrom, and R. Bremner for providing SFV vectors and help with generating SFV particles. We thank R. Tsien (Howard Hughes Medical Institute) for providing monomeric red fluorescent protein DNA and S. Carbonetto and D. van Meyel for valuable comments regarding this manuscript.

Correspondence should be addressed to Dr. Keith K. Murai, Centre for Research in Neuroscience, Montreal General Hospital, 1650 Cedar Avenue, L7-212, Montreal, Quebec, H3G 1A4, Canada. E-mail: keith.murai@mcgill.ca. DOI:10.1523/JNEUROSCI.1302-06.2006

Copyright $\odot 2006$ Society for Neuroscience $\quad$ 0270-6474/06/268881-11\$15.00/0 correlate with long-term potentiation (LTP) and long-term depression, respectively (Matsuzaki et al., 2004; Nagerl et al., 2004; Zhou et al., 2004). Thus, proper control over synaptic structure may be essential for the coding of information in the brain. Consistent with this, abnormalities in spine density and architecture are associated with neurological diseases, especially those involving mental retardation (Kaufmann and Moser, 2000).

Surprising roles for glial cells in synapse development and physiology have recently emerged (Castonguay et al., 2001; Haydon, 2001; Volterra and Meldolesi, 2005). During synaptogenesis, glial cells release cholesterol and thrombospondins to increase synapse number and maturation (Ullian et al., 2001; Christopherson et al., 2005). Later, astrocytes define extracellular neurotransmitter levels, buffer the extracellular milieu, and propagate $\mathrm{Ca}^{2+}$ signals (Haydon, 2001). Astrocytes also contain machinery for vesicular-mediated release of "gliotransmitters," including glutamate, ATP, and D-serine, which modulate synaptic function (Volterra and Meldolesi, 2005; Panatier et al., 2006). Despite our knowledge of the physiological connections between astrocytes and neurons, little is known about their morphological interactions. At the ultrastructural level, astrocytes and synapses are intimately associated in the hippocampus (Ventura and Harris, 1999). Astrocytic processes also prefer to localize near dendritic spines versus presynaptic terminals (Lehre and Rusakov, 2002), indicating a complex interplay between astrocytes and the postsynaptic surface.

Intriguingly, astrocytes display morphological rearrangements in acute brainstem and organotypic hippocampal slices 
(Hirrlinger et al., 2004; Benediktsson et al., 2005; Haber and Murai, 2006). However, the details behind this motility and, importantly, its relationship to the structural plasticity of spines remain to be clarified. Here we characterize astrocyte-synapse interactions in situ by simultaneous manipulation of neurons and glia in hippocampal slices. Astrocytes show striking remodeling behavior over minutes, display elaborate process extension and retraction, and likely use the actin cytoskeleton for motility. Furthermore, astrocytic processes show cooperative motility with spines and can rapidly redefine their proximity to the synapse. These studies unveil the dynamic exchange that occurs between astrocytes and central synapses and suggest that concerted structural plasticity of both neurons and glia plays a role in synaptic function.

\section{Materials and Methods}

Semliki Forest virus plasmid construction and virus preparation. For expressing fluorescent proteins in hippocampal slices, Semliki Forest virus (SFV) constructs were created (Ehrengruber et al., 1999). Genes for farnesylated enhanced green fluorescent protein (EGFPf) (Clontech, Cambridge, UK) and farnesylated red fluorescent protein (RFPf) were cloned into Semliki Forest virus vectors. The genes were cloned into SFV PD (Lundstrom et al., 2003) for expression in neurons or into SFV A7 (Ehrengruber et al., 2003) for expression in astrocytes after the viral nonstructural proteins and the subgenomic promoter. Farnesylated monomeric RFP (mRFP) was created by cloning the farnesylation sequence of Ha-Ras onto the C terminus of mRFP (from R. Tsien, University of California, San Diego, La Jolla, CA) in pcDNA3. Membrane targeting was confirmed by transfecting the construct into COS7 cells and comparing the subcellular localization with cytoplasmic mRFP. Significant improvement over mRFP in the delineation of fine cellular processes was achieved with RFPf. Viral particles were created by cotransfecting SFV vectors with a viral packaging vector into baby hamster kidney cells (DiCiommo and Bremner, 1998). At $72 \mathrm{~h}$ after transfection, cell media were collected and purified on a sucrose gradient ( 20 and $55 \% \mathrm{w} / \mathrm{v}$ ) by ultracentrifugation. Fractions containing viral particles were then diluted with PBS and concentrated using a high-molecular-weight cutoff filter column (Amicon, Beverly, MA) and low-speed centrifugation. Viral particles were resuspended in ice-cold PBS and activated by chymotrypsin $(10 \mathrm{mg} / \mathrm{ml})$, followed by aprotinin $(10 \mathrm{mg} / \mathrm{ml})$ treatment. Viruses were injected into hippocampal slices with a Picospritzer (General Valve, Fairfield, NJ).

Tissue preparation and hippocampal slice culture. For diolistic labeling of neurons and astrocytes in the hippocampus, $300 \mu \mathrm{m}$ hippocampal slices were created using a McIllwain tissue chopper (Stoelting, Kiel, WI) and immediately fixed in $4 \%$ paraformaldehyde $/ 0.1 \mathrm{M} \mathrm{PO}_{4}{ }^{2-}$ buffer for $30 \mathrm{~min}$. Tungsten particles $(1.3 \mu \mathrm{m})$ (Bio-Rad, Hercules, CA) carrying the lipophilic dyes DiI or DiO (Invitrogen, Carlsbad, CA), were prepared as described previously (Gan et al., 2000). Particles were coated onto the inner lining of plastic cartridges, loaded into a Helios Gene Gun (Bio$\mathrm{Rad})$, and propelled into hippocampal slices at 120 psi. The dyes were allowed to transport within slices for $16 \mathrm{~h}$ in fixative before imaging by confocal microscopy.

Organotypic hippocampal slices were prepared as described previously (Stoppini et al., 1991; Murai et al., 2003). Briefly, $300 \mu \mathrm{m}$ slices from postnatal day 6-7 mouse pups were made using a McIllwain tissue chopper (Stoelting) and transferred onto semiporous tissue culture inserts $(0.3 \mu \mathrm{m}$ pore size; Millipore, Bedford, MA) containing media ( $50 \%$ minimum essential medium, 25\% horse serum, 25\% HBSS, $6.5 \mathrm{mg} / \mathrm{ml}$ D-glucose, and $0.5 \%$ penicillin/streptomycin, $\mathrm{pH} \sim 7.2$ ). Media were replaced every $2 \mathrm{~d}$, and slices were cultured for $1-3$ weeks before viral gene delivery. At $16-20 \mathrm{~h}$ after infection, slices were either fixed for static confocal imaging or used for time-lapse imaging experiments.

Immunofluorescence on hippocampal slices. Immunofluorescence on hippocampal slices was performed as described previously (Murai et al., 2003). Briefly, membranes containing slices were cut out from plastic inserts, fixed in $4 \%$ paraformaldehyde/0.1 $\mathrm{M} \mathrm{PO}_{4}{ }^{2-}$ buffer for $30 \mathrm{~min}$, washed for $5 \mathrm{~min}$ in Tris-buffered saline (TBS), blocked in 10\% goat serum (Jackson ImmunoResearch, West Grove, PA) in TBS containing $0.1 \%$ Triton X-100 (TBS-T), and incubated with primary monoclonal antibodies overnight. The following antibodies were used: GFAP (monoclonal; Sigma, St. Louis, MO); neurofilament (monoclonal; from V. Lee, University of Pennsylvania, Philadelphia, PA); NG2 (rabbit polyclonal; Chemicon, Temecula, CA); cyclic nucleotide $3^{\prime}$-phosphodiesterase (CNP) (monoclonal; Sigma); MBP (monoclonal; Sternberger Monoclonals, Lutherville, MD); and glutamate transporter-1/excitatory amino acid transporter-2 (GLT-1/EAAT-2) (rabbit polyclonal; Santa Cruz Biotechnology, Santa Cruz, CA). The next day, slices were washed four times for $30 \mathrm{~min}$ each with TBS-T before incubation with either goat antimouse or anti-rabbit Alexa-568 or Alexa-647 (Invitrogen, Carlsbad, CA). Slices were then washed four times for $30 \mathrm{~min}$ and then imaged with confocal microscopy. Immunostaining using a monoclonal antibody against synaptophysin (Sigma) was essentially the same as described above, but $0.2 \%$ Triton $\mathrm{X}-100$ was used in the blocking and washing steps.

Static and time-lapse confocal imaging. Static confocal imaging was performed using an Ultraview spinning disk confocal system (PerkinElmer, Wellesley, MA) connected to an Eclipse TE2000 (Nikon, Tokyo, Japan) equipped with a $60 \times$ oil immersion lens ( 1.25 numerical aperture with $1.5 \times$ optical zoom; Nikon). $Z$-stacks were collected using MetaMorph imaging software (Molecular Devices, Palo Alto, CA) and deconvoluted in three dimensions using Autodeblur software (AutoQuant, Media Cybernetics, Silver Spring, MD). A calculated pointspread function optimized for spinning disk confocal microscopy was used. For time-lapse imaging, an Ultraview spinning disk system was connected to a Leica (Nussloch, Germany) DMS upright microscope equipped with a $63 \times$ extra-long working distance lens (Leica). Hippocampal slices were secured in a heated electrophysiology chamber (Warner Instruments, Hamden, CT) and perfused with oxygenated artificial CSF (ACSF) (125 mu NaCl, $3 \mathrm{~mm} \mathrm{KCl}, 1.25 \mathrm{~mm} \mathrm{NaH}_{2} \mathrm{PO}_{4}, 26 \mathrm{~mm}$ $\mathrm{NaHCO}_{3}, 2.4 \mathrm{M} \mathrm{CaCl}_{2}$, and $10 \mathrm{~mm}$ glucose, $95 \% \mathrm{O}_{2} / 5 \% \mathrm{CO}_{2}$ ) that was heated to $32-34^{\circ} \mathrm{C}$ (Warner Instruments). $Z$-stacks were taken at $3 \mathrm{~min}$ intervals unless specified otherwise using MetaMorph software. In pharmacology experiments, baseline time-lapse images were collected before treatment of slices with ACSF containing cytochalasin-D ( $5 \mu \mathrm{M}$; Sigma) or bicuculline methochloride ( $20 \mu \mathrm{M}$; Sigma). Imaging was resumed 3 min after the start of the drug treatment.

Measurements and quantifications. For quantification of spine and astrocytic process motility, a motility index was computed for astrocytic processes adjacent to spines similar to Dunaevsky et al. (1999). Images were first thresholded in NIH ImageJ. Areas of the spines were measured as was the closest glial process adjacent to the spine within sequential maximum projected frames of the time-lapse series. The average area was then computed. Total accumulated area for neuron and glial movements was performed by summating all of the frames in a time-lapse series for each channel and measuring the maximum area. The motility index was computed by subtracting the smallest area from the total accumulated area and dividing by the average area. A total of 71 astrocyte-spine pairs taken over seven experiments were analyzed for Figure $7 a-d$. Because significant differences were not observed between slices kept $1-3$ weeks in culture, the data were pooled for quantification purposes. In Figure $7 b$, a cutoff was used to separate astrocytic process motilities that were $50 \%$ larger or smaller than their corresponding spine motilities. In Figure $7 c$, which shows percentages of coordinated, noncoordinated, and opposite changes in astrocyte/spine morphology, a 10\% increase or decrease in astrocyte or spine area between the first and last time point of an imaging series was considered growth or retraction, respectively. Spines and astrocytic processes that did not show a more than $10 \%$ change were considered stable. Thus, instances in which astrocytes and spines both showed $>10 \%$ growth or retraction were considered coordinated. The movements were also considered coordinated if both showed $<10 \%$ change in size. Instances in which the astrocytic process and spine showed reversed growth or retraction from each other were considered to be opposites of each other. All other instances were considered to be noncoordinated. In Figure 7e, 20 dendritic spines and 25 astrocytic pro- 

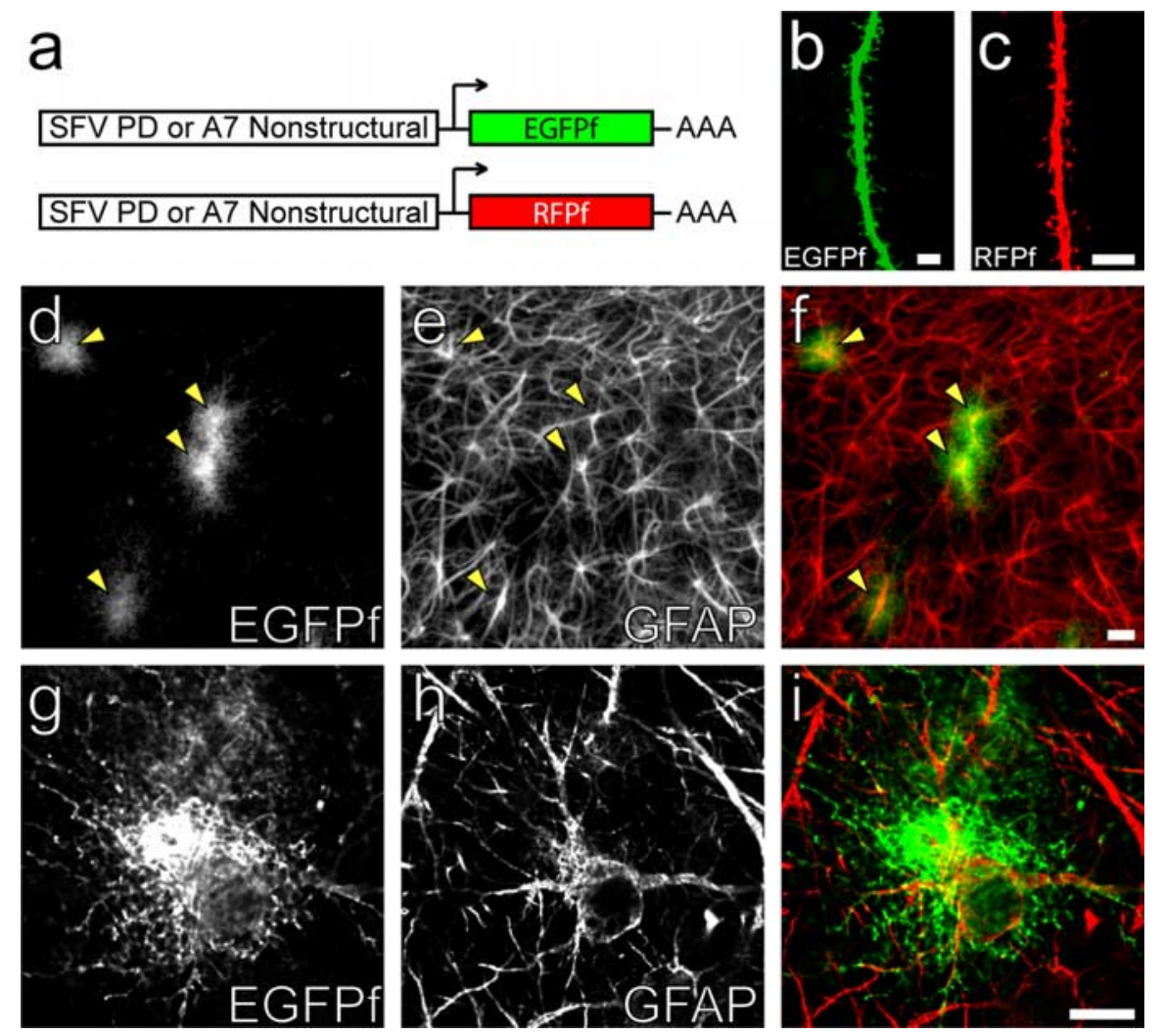

Figure 1. Targeting complex neuron-glial interactions in the hippocampus using organotypic hippocampal slices and viral gene delivery. $\boldsymbol{a}$, SFV constructs used in the study. EGFPf or RFPf were cloned after the genes encoding viral nonstructural proteins and a subgenomic promoter (arrow) on SFV plasmids. $\boldsymbol{b}, \boldsymbol{c}$, Confocal images showing examples of apical dendrites with prominent spines on CA1 pyramidal neurons infected with SFV PD EGFPf ( $\boldsymbol{b}$, green) or SFV PD RFPf (c, red). $\boldsymbol{d}$ - $\boldsymbol{f}$, Low-magnification epifluorescence images showing the selectivity of SFV A7 for protoplasmic astrocytes in the stratum radiatum of area CA1. Infected astrocytes expressing EGFPf ( $\boldsymbol{d}$, green; arrowheads) are labeled for GFAP (e, red). $\boldsymbol{g}-\boldsymbol{i}$, Single-plane confocal images of an SFV A7-infected astrocyte showing EGFPf expression ( $\boldsymbol{g}$, green) and GFAP immunoreactivity $(\boldsymbol{h}$, red). Note how the GFAP immunolabeling only labels the main processes of the infected protoplasmic astrocyte, whereas EGFPf reveals the fine processes (i). Scale bars: $\boldsymbol{b}, \boldsymbol{c}, 5 \mu \mathrm{m} ; \boldsymbol{d}-\boldsymbol{i}, 10 \mu \mathrm{m}$.

cesses over two experiments were analyzed. In Figure $7 f, 31$ dendritic spines and 49 astrocytic processes over two experiments were analyzed.

Three-dimensional reconstructions. For Figures $3, g$ and $h$, and 4 and supplemental Figure 2 (available at www.jneurosci.org as supplemental material), deconvoluted confocal $Z$-stacks were rendered in three dimensions using IMARIS 4.5.2 (Bitplane, Zurich, Switzerland). For Figure 4 and supplemental Figure 2 (available at www.jneurosci.org as supplemental material), synaptophysin and GLT-1/EAAT-2 proximity to dendrites were determined by masking the synaptophysin or GLT-1/EAAT-2 channel with a second three-dimensional rendering of the dendritic segment extending $\sim 0.5 \mu \mathrm{m}$ from the surface shown (using IMARIS 4.5.2; Bitplane), leaving only those punctae within close proximity of the dendritic spines and shaft.

Three-dimensional quantifications. For quantification of astrocytic process extension and retraction (see Fig. 5), individual astrocytic processes from deconvoluted $Z$-stacks were isolated using contour surfaces (IMARIS MeasurementPro; Bitplane) and rendered in three dimensions (IMARIS 4.5.2; Bitplane). Volume changes were plotted over time.

For quantifications in Figure 8, dendritic segments containing spines that were encompassed within an astrocyte domain were chosen for analysis (with the assumption that each astrocyte occupies its own territory) (see Fig. 2). For three-dimensional quantification of dendritic spineastrocyte profiles, contour surfaces (IMARIS MeasurementPro; Bitplane) were first used to separate dendritic shafts from spines or spine heads. Three-dimensional surfaces of spines were generated and used to mask the surrounding astrocytic channel, leaving only the overlap between spines and astrocytic processes (IMARIS 4.5.2; Bitplane). Surfaces of the overlap, or astrocytic profile, of each spine were generated and used to track volume changes. To control for artifacts caused by minor fluctuations in signal intensity or sample photobleaching, multiple dendritic shaft segments along each quantified region were used to adjust surface thresholds for spine and astrocytic profile volume rendering at each time point (supplemental Fig. 1, available at www. jneurosci.org as supplemental material). We assumed that dendritic shaft segments were stable structures over the imaging period. In Figure $8 b$, percentage volume change was determined by comparing the minimum and maximum value of both spine and astrocytic profile volume over six time points at $3 \mathrm{~min}$ intervals ( 15 min total; $n=32$ pairs of astrocytes and spines over three experiments taken at 3 weeks in vitro).

\section{Results}

Comanipulation of neurons and astrocytes in hippocampal slices Recent studies have described dynamic morphological changes at synapses in the nervous system with particular emphasis on postsynaptic dendritic spines (Dailey and Smith, 1996; Fischer et al., 1998; Dunaevsky et al., 1999). However, little is known about the morphological properties of astrocytic processes that are juxtaposed to synapses and serve to modulate synaptic function. This is, in part, attributable to the lack of system that can preserve the three-dimensional architecture of astrocytes near synapses and a reliable method to express proteins in astrocytes or neurons. We overcame these issues using organotypic hippocampal slices that can be infected efficiently and selectively with viral vectors. We made use of two Semliki Forest viral strains that primarily target neurons (SFV PD) or glial cells (SFV A7) in hippocampal slices (Ehrengruber et al., 2003; Lundstrom et al., 2003). Viral particles were created that deliver genes for either EGFPf or RFPf. The expression and membrane tethering of these fluorescent proteins allowed enhanced visualization of fine cellular processes including dendritic spines on neurons and highly ramified astrocytic processes (Fig. 1).

To deliver genes to astrocytes, we modified a nonvirulent strain of SFV that is known to infect glial cells (SFV A7) (Ehrengruber et al., 2003). To confirm that the SFV A7 strain was targeting astrocytes in our hippocampal slices, we injected slices with the virus at $7 \mathrm{~d}$ in vitro, allowed $16-20 \mathrm{~h}$ for expression of fluorescent proteins, and subjected the slices to immunofluorescence using antibodies against several marker proteins. Protoplasmic cells expressing EGFPf were labeled with GFAP antibodies in all instances, confirming their identity as astrocytes (Fig. 1). Imaging the cells at higher magnification showed that EGFPf extensively labeled the membrane of astrocytes including their fine processes, whereas GFAP was only localized to the main processes and central region of astrocytes (Fig. $1 \mathrm{~g}-i$ ). This is consistent with reports showing that GFAP is only found in the main astrocytic stalks (Bushong et al., 2002). The targeted astrocytes were also positive for the glial glutamate transporter (GLT-1/ EAAT2) (supplemental Fig. 2, available at www.jneurosci.org as 
supplemental material), indicating that viral infection and expression of membrane-bound GFP did not perturb the basic molecular properties of these cells. It further suggests that the targeted astrocytes in our system may be of the subtype that is important for glutamate reuptake (Volterra and Meldolesi, 2005). GLT-1 was found to be clustered on the surface of these cells as has been reported previously using immunostaining of cultured astrocytes (Zhou and Sutherland, 2004). The EGFPf labeled protoplasmic astrocytes were negative for other markers including neurofilament, NG2, CNP, and MBP (data not shown). We found that protoplasmic astrocytes expressing EGFPf were mainly confined to regions below the surface of hippocampal slices in which the virus injections were made. Similar to Benediktsson et al. (2005), we did note the occasional presence of more simplified and thickly branched GFAP-positive cells expressing EGFPf at the superficial layers of the slice (top 10-20 $\mu \mathrm{m})$. These cells were phenotypically different from protoplasmic astrocytes within the slice and may represent "reactive" glia. However, because our virus injections were made below the superficial layers $(\sim 100 \mu \mathrm{m}$ below the surface of the slice), we mainly infected protoplasmic astrocytes that show a highly ramified morphology (Bushong et al., 2002; Ogata and Kosaka, 2002). Thus, this system can be efficiently used to delineate and monitor both neuronal and protoplasmic astrocyte morphology.

Preservation of complex three-dimensional glial-glial and neuron-glial interactions in organotypic hippocampal slices We observed that EGFPf- or RFPf-expressing astrocytes in hippocampal slice cultures retained their complex threedimensional morphologies, similar to what has been described with Golgi labeling or single-cell injections of fluorescently labeled probes into fixed brain tissue (Bushong et al., 2002; Ogata and Kosaka, 2002). Furthermore, astrocytes in hippocampal slice culture also occupied highly segregated domains that were not evident when cells were labeled for GFAP (Fig. $1 d-f$ ). This could be visualized more effectively by coinfecting adjacent astrocytes with viruses expressing either EGFPf or RFPf in the stratum radiatum of hippocampal area CA1 (Fig. $2 d-f$ ). Neighboring astrocytes showed only limited overlap at the periphery of their domains. This domain segregation was similar to the "tiling" observed when we used diolistics to label adjacent astrocytes in area CA1 with DiI and DiO in fixed adult hippocampal tissue (Fig. $2 a-c$ ). These results are also consistent with studies that have characterized the spatial relationship of astrocytes using single-cell injections of dyes into adjacent cells in fixed tissue (Bushong et al., 2002, 2004). The maintenance of this domain segregation indicates that cellular programs or interactions that establish the boundaries between neighboring astrocytes remain intact in hippocampal slice culture.

We next turned our analysis to complex neuron-astrocyte interactions in the stratum radiatum of area CA1. Intricate threedimensional interactions between astrocytes and neurons have been described using serial electron microscopy (Ventura and Harris, 1999). Despite the fine resolution of this methodology, it is limited to fixed tissue and requires serial sectioning coupled
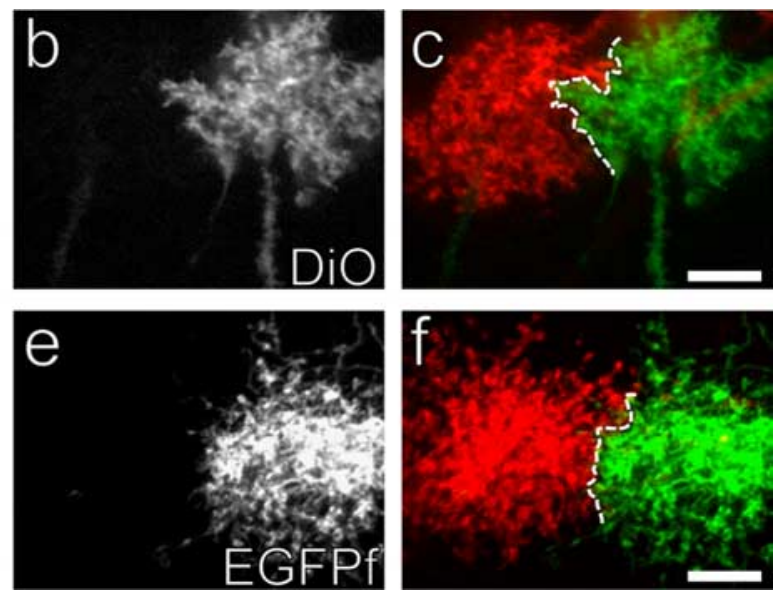

RFPf

Figure 2. Astrocytic elaboration and domain spacing in area CA1 of the adult mouse hippocampus and in organotypic slice culture. $\boldsymbol{a}-\boldsymbol{c}$, Diolistic labeling of protoplasmic astrocytes in fixed hippocampal tissue. Maximum projections of confocal images of neighboring astrocytes labeled with Dil $(\boldsymbol{a}, \mathrm{red})$ and Di0 $(\boldsymbol{b}$, green) reveals their distinct domain spacing. $\boldsymbol{d}-\boldsymbol{f}$, Astrocytes in ip cytes expressing RFPf (d, red) and EGFPf (e, green) after 1 week in vitro. The dashed lines in c and $\boldsymbol{f}$ indicate the boundary between s, $10 \mu \mathrm{m}$

with electron microscopy. We were interested in establishing a system for simultaneously imaging juxtaposed neurons and astrocytes with dual-color confocal microscopy in live cells. Using combined SFV PD and A7 virus injections, we infected astrocytes that were adjacent to neurons and observed this complex morphological interplay (Fig. 3) (Ventura and Harris, 1999). Hippocampal slices were grown for 7-21 d in vitro, and SFV PD and SFV A7 viral particles were coinjected into the neuropil of area CA1. After 16-20 h of expression, we fixed the tissue and prepared the slices for microscopy. In each slice, we could identify several sites of neuron-glial interaction. Using confocal $Z$-series imaging, we obtained stacks of images of RFPf-labeled dendrites juxtaposed to EGFPf-labeled astrocytic processes and analyzed them by computer-based three-dimensional reconstruction. Similar to the observations using serial electron microscopy, astrocytes and dendrites/spines showed highly complex associations (Fig. 3g). In many instances, astrocytes extended fine processes toward spines. In some cases, astrocytic processes fully encapsulated spines. The complex three-dimensional organization of spine-astrocyte interactions were found to be similar to experiments using diolistic labeling of fixed adult hippocampal slices (Fig. $3 a-c$ ). Interestingly, dendrites in fixed tissue and in organotypic cultures extend freely through the domain of a single astrocyte and presumably into that of a neighboring astrocyte, ignoring those signals responsible for astrocytic segregation. Several attempts to label astrocytes overlapping with CA1 pyramidal cell dendrites in organotypic cultures using biolistic gene gun transfections gave unreliable results because of the low frequency of transfecting nearby cells with gold particles bearing different plasmids.

We confirmed that the majority of dendritic spines in our slice culture system were associated with presynaptic terminals (Fig. 4). After 1 and 3 weeks in culture, we subjected slices to immunolabeling with anti-synaptophysin antibodies. As depicted in Figure 4, the majority of spines showed synaptophysin labeling adjacent to RFPf-labeled dendritic spines, indicating that most of the spines have presynaptic innervation. Altogether, these results indicate that many of the properties of astrocyte-astrocyte, neuron-astrocyte, and neuron-neuron interactions are maintained and easily visible in three dimensions in organotypic slice culture. 

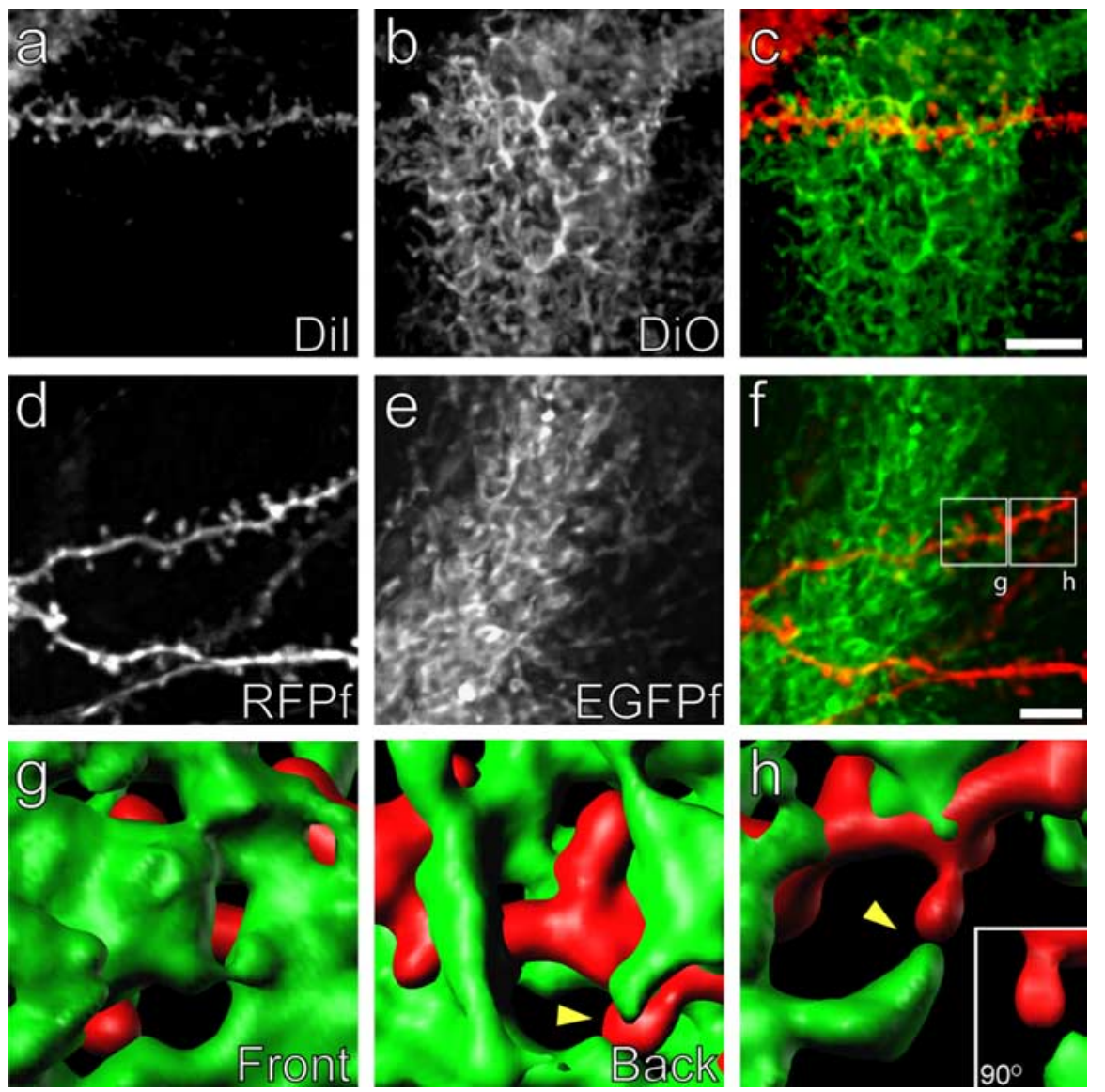

Figure 3. Complex neuron-glial interactions in the hippocampal CA1 neuropil. $\boldsymbol{a}-\boldsymbol{c}$, Confocal maximum projections of a Dil-labeled apical dendrite from a CA1 pyramidal cell $(\boldsymbol{a}$, red) extending through a Di0-labeled astrocyte domain $(\boldsymbol{b}$, green) in a fixed slice of the adult hippocampus. Areas of overlap show the complexity of neuron-glial interactions. $\boldsymbol{d}-\boldsymbol{f}$, Confocal maximum projections of a dendrite from an SFV PD RFPf-infected CA1 pyramidal neuron (d, red) projecting through an SFV A7 EGFPf-infected astrocyte domain ( $\boldsymbol{e}$, green) after 1 week in vitro. $\boldsymbol{g}, \boldsymbol{h}$, Three-dimensional reconstructions of the boxed areas in $\boldsymbol{f}$, demonstrating the complex interplay between astrocytic processes and dendritic spines. Inset in $\boldsymbol{h}$ shows the neuron-glial association rotated $90^{\circ}$. Arrowheads show examples of astrocytic processes extending toward spines. Scale bars, $5 \mu \mathrm{m}$.

cesses consolidated into a single one. These results demonstrate that astrocytic processes can undergo dramatic changes in their structural organization, a behavior that may enable them to relocate their processes around synapses.

\section{Cooperative astrocyte-dendritic spine motility}

We next were interested in determining the dynamic relationship between astrocytes and dendritic spines. We focused our attention on postsynaptic spines because they have been shown to have a wide range of motile behaviors (Dailey and Smith, 1996; Fischer et al., 1998; Dunaevsky et al., 1999) and astrocytes appear to be preferentially associated with them (Lehre and Rusakov, 2002). To investigate the dynamics between astrocytic processes and dendritic spines, we simultaneously injected slices with SFV PD and A7 and coimaged dendritic spines and astrocytic processes over time. We found that astrocytes extended and retracted processes near dendritic spines over the course of minutes (Fig. 6) (supplemental movies 2, 3 , available at www.jneurosci.org as supplemental material). In some instances, the astrocyte-spine interactions were stable, whereas in others, striking changes in organization were observed. Using threedimensional reconstruction of $Z$-stacks, we confirmed that astrocytic membranes are closely associated with and appear to contact dendritic spines. After calculating a motility index for astrocytic processes adjacent to dendritic spines, we found that, on average, astrocyte motility was

\section{Astrocytes display a wide range of dynamic motility}

Most reports have described the structural properties of glial cells in dissociated cultures in which astrocytes typically exhibit a simplified two-dimensional cellular morphology. Thus, dissociated astrocytes do not fully mimic the features of astrocytes in vivo. This is in contrast to astrocytes in hippocampal slice cultures, in which the cells show complex three-dimensional morphology (Benediktsson et al., 2005). We were interested in determining whether astrocytic processes in our system undergo dynamic changes and, if so, what were the properties of the remodeling. Slices were cultured for 1-3 weeks before injection with SFV A7 delivering EGFPf. Sixteen to $20 \mathrm{~h}$ later, the slices were transferred to an imaging chamber, perfused with oxygenated ACSF (32$34^{\circ} \mathrm{C}$ ), and imaged using time-lapse confocal microscopy. Protoplasmic astrocytes showed remarkably dynamic behavior that occurred over short time periods (minutes). We observed different types of motility throughout the astrocytic domain (Fig. 5) (supplemental movie 1, available at www.jneurosci.org as supplemental material). In many cases, widespread extension and retraction (sometimes over distances of $>5 \mu \mathrm{m}$ ) occurred during a 15-30 min imaging period. Thin, lamellipodial-like processes were also found that showed a continuous "morphing" similar to what has been described for the head portion of dendritic spines (Dunaevsky et al., 1999). In other cases, single processes split or two pro- significantly higher than the motility of the corresponding dendritic spine $(p<0.007)$ (Fig. 7). Plotting spine motility versus astrocyte motility showed three main populations of associations between spine and astrocyte motility. In 36 of 71 cases (51\%), astrocytic processes exhibit a similar level of motility as their adjacent spine. However, in 23 of 71 cases (32\%), astrocytic processes showed much higher motility relative to the spine, whereas in only 12 of 71 cases (17\%), spines showed higher motility than the adjacent astrocytic process.

In most cases, the relative trajectories of spine and astrocyte movements were not associated. However, astrocytic processes rapidly reconfigured their location around spine heads (Fig. 6) (supplemental movies 2, 3, available at www.jneurosci.org as supplemental material). Despite the lack of coordinated directional movement, $50 \%$ of spines showed cooperative changes in size (in terms of growth, retraction, or stability) with astrocytic processes, whereas $27 \%$ of spines did not show cooperative changes, and $23 \%$ spines showed opposite changes in size relative to astrocytic processes. Thus, astrocytic processes mainly show cooperative growth processes with spines, indicating a positive relationship between astrocyte and spine growth/retraction.

Dendritic spines are known to use actin-based cytoskeletal rearrangements for their motility (Fischer et al., 1998; Matus, 2000). We were interested in whether astrocyte dynamics also 
a

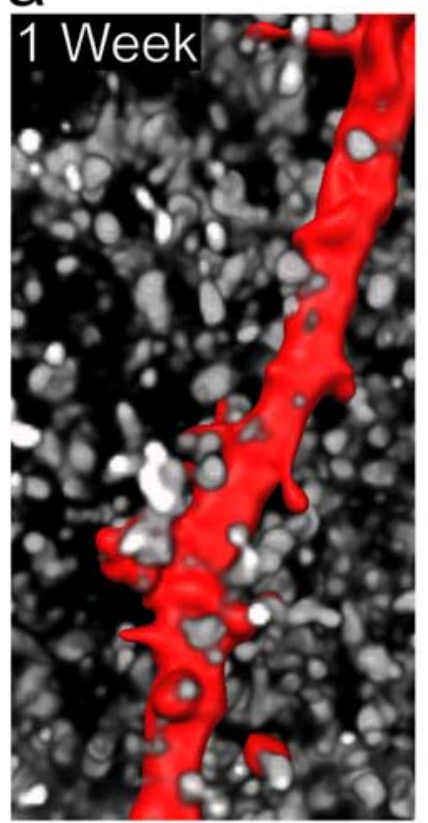

b

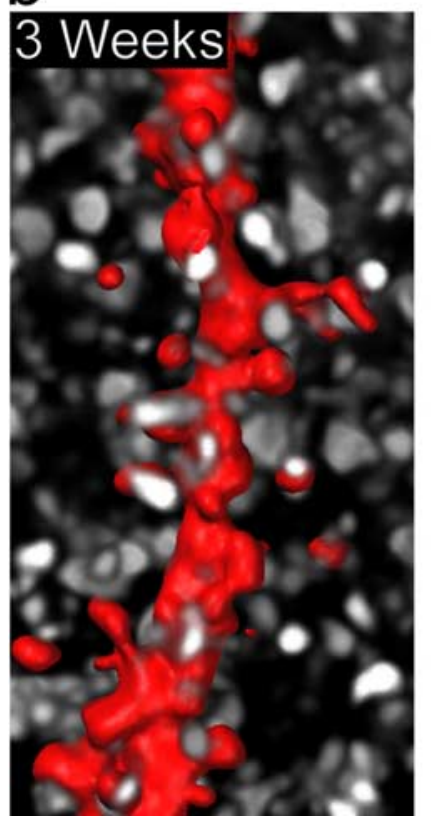

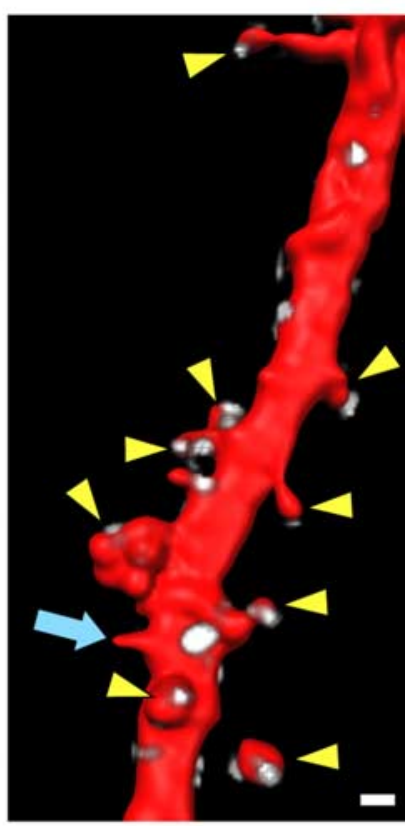

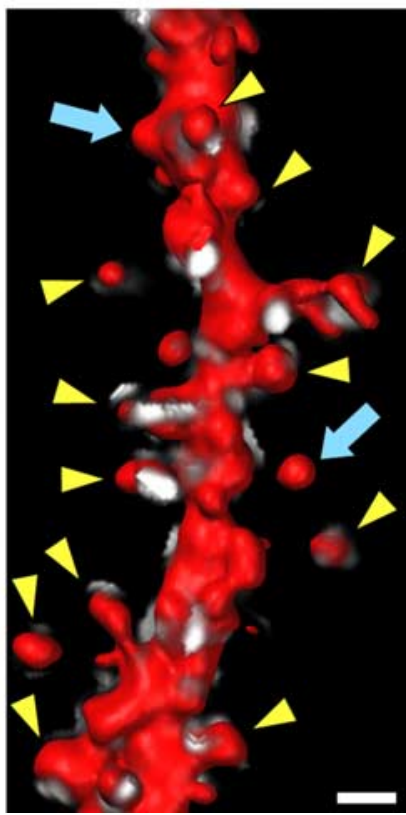

Figure 4. Dendritic spines are innervated in hippocampal slice culture. $\boldsymbol{a}, \boldsymbol{b}$, SFV PD RFPfinfected slices at 1 and 3 weeks in vitro and immunolabeled for synaptophysin (white) to label presynaptic terminals. At both time points, the majority of CA1 pyramidal cell dendritic spine heads (red) are innervated by presynaptic terminals (arrowheads), whereas only a small proportion are not (arrows). Spines that show association of synaptophysin punctae on the neck are not labeled. Shown are images of the synaptophysin labeling before and after performing a three-dimensional "masking" procedure to preserve only the synaptophysin punctae that are associated with the dendrite (see Materials and Methods). Scale bars, $1 \mu \mathrm{m}$.

used actin filament turnover. Using spine motility as an internal control, we found that application of cytochalasin-D to the perfusion media significantly reduced astrocytic process motility. Figure $7 e$ shows the reduction in motility indexes of spines and astrocytic processes before and after application of cytochalasin-D (spines, $p<0.003$, paired Student's $t$ test; astrocytic processes, $p<0.006$, paired Student's $t$ test). These results suggest that astrocytic rearrangements in hippocampal slices use

modifications to the actin cytoskeleton. However, we cannot rule out the possibility that blocking spine motility with cytochalasin-D indirectly influences astrocytic motility. Reports have also shown that disruption of actin filament reorganization with cytochalasin-D or latrunculin can affect synaptic transmission, especially after prolonged administration (i.e., >30 min) (Kim and Lisman, 1999; Korkotian and Segal, 2001). This, in turn, may affect the motility of astrocytic processes.

To test whether general modifications to neural activity affect both spine and astrocytic motility, we calculated motility indices before and after application of the $\mathrm{GABA}_{\mathrm{A}}$ receptor antagonist bicuculline. We used bicuculline to globally increase neural activity in slices because a previous study has shown that it can stabilize rapid changes in dendritic spine shape (Korkotian and Segal, 2001). This is in contrast to studies that have applied $\mathrm{KCl}$ (to generally depolarize neurons) or TTX (to block action potentials), which do not appear to significantly influence spine motility in slices (Dunaevsky et al., 1999; Oray et al., 2006). To examine the effects of bicuculline application, we initially measured spine and astrocytic motility under control ACSF conditions. Bicuculline was then introduced into the ACSF, and, 3 min later, imaging was resumed and motility indices were calculated. We found that bicuculline significantly reduced spine motility by $28 \%$ ( $p=$ 0.002 , paired Student's $t$ test) (Fig. $7 f$ ). This is consistent with reports that have shown that glutamatergic signaling through AMPA receptors reduces spine motility (McKinney et al., 1999; Fischer et al., 2000). In contrast, bicuculline did not significantly change the overall motility of astrocytes, only reducing the average motility index by $13 \%$ ( $p>0.05$, paired Student's $t$ test). However, we did notice that bicuculline application caused a $>10 \%$ reduction in spine and astrocytic motility in 65 and $55 \%$ of the cases, respectively. Thus, decreasing the inhibitory tone in hippocampal slice cultures with bicuculline results in the stabilization of spines but does not significantly reduce overall astrocytic motility.

\section{Continuous remodeling of astrocytic association with dendritic spines}

An important function of astrocytes is to regulate the synaptic microenvironment by removing neurotransmitter from the extracellular space and releasing neuroactive substances (i.e., glutamate, ATP, and D-serine). This function is likely influenced by the location of fine astrocytic extensions near the synapse. We were interested in determining the degree to which astrocyte proximity could be reorganized around spines over time. We infected slices with viruses after 3 weeks in culture and subjected them to time-lapse confocal imaging. Sites of interaction between spines and astrocytic processes were reconstructed in three dimensions, and their relationship was examined (Fig. $8 a$ ). To reduce the possibility that spines were being contacted by multiple astrocytes, we chose spines that were localized within a single astrocyte domain. We observed that larger spines were more stable, showing lower spontaneous volume changes over time (Fig. $8 b$ ). This is consistent with our motility index analysis in which spine motility decreased with increased spine area and with in vivo data showing that larger spines are, in general, more stable (Grutzendler et al., 2002; Holtmaat et al., 2005). Interestingly, the degree of proximity of astrocytic profiles to spines rapidly changed over the course of minutes. Figure $8 a$ shows an example of a relatively stable mushroom-shaped spine and the change in astrocyte juxtaposition during a 15 min imaging period. Plotting the maximum volume change of the astrocyte profile over the imaging period versus the average volume of the spine showed 

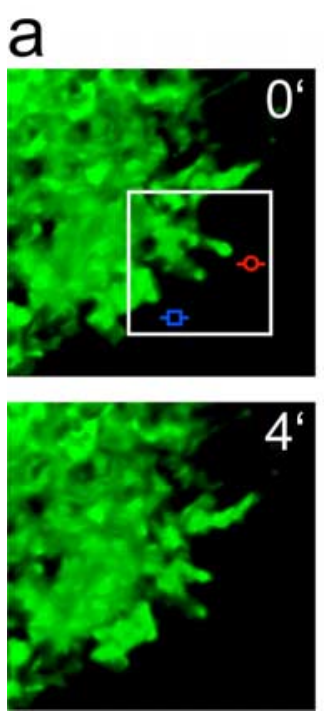

b
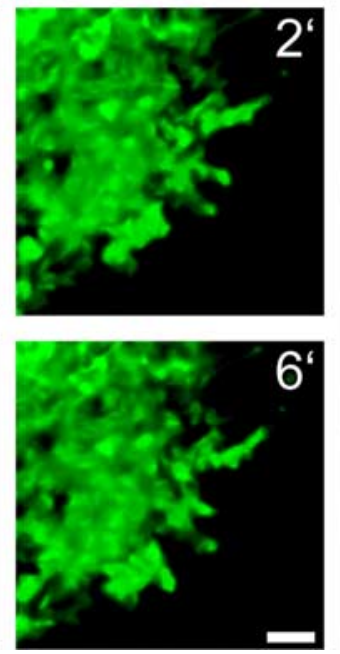
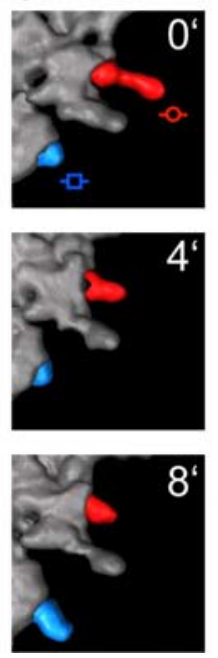
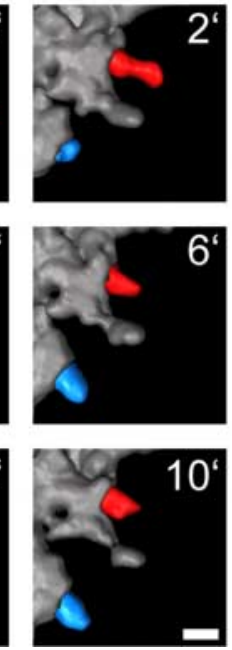

C

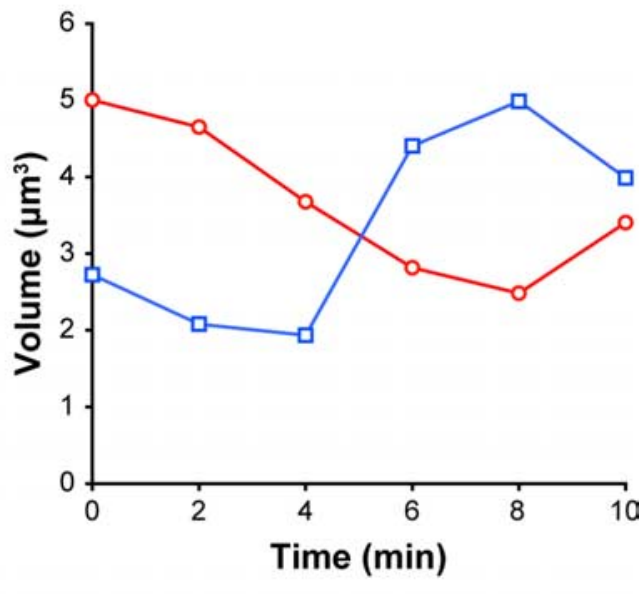

Figure 5. Astrocytes undergo rapid structural modifications. $\boldsymbol{a}$, An example showing astrocytic process retraction and extension over a 10 min imaging period (confocal maximum projections of the first 4 time points are shown). $\boldsymbol{b}$, Three-dimensional reconstructions of the area outlined in $\boldsymbol{a}$ over the 10 min period. $\boldsymbol{c}$, Volume measurements of retracting (red circles) and extending (blue squares) processes plotted over time. Scale bars: $\boldsymbol{a}, 10 \mu \mathrm{m} ; \boldsymbol{b}, 4 \mu \mathrm{m}$.
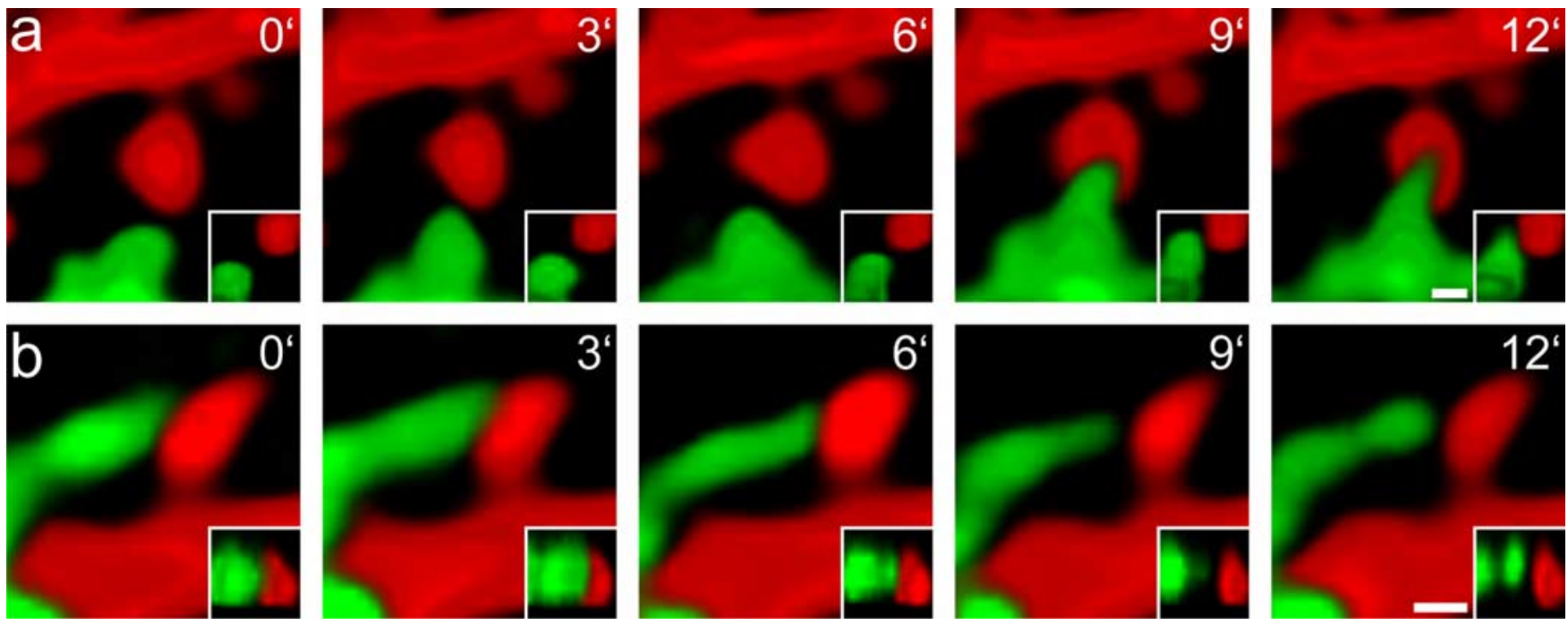

Figure 6. Astrocyte-spine interactions show dynamic structural interplay. $\boldsymbol{a}$, Example of an astrocytic process (green) extending toward a dendritic spine (red). $\boldsymbol{b}$, Example of an interaction that is lost between a dendritic spine (red) and a retracting astrocytic process (green). Insets show $90^{\circ}(\boldsymbol{a})$ or top-down (b) views of the astrocyte-spine interactions. Scale bars, $1 \mu \mathrm{m}$.

that spines with greater volume have more stable contacts with astrocytic processes (Fig. $8 b$ ). These results suggest that the stability of glial associations with the postsynaptic surface can differ based on spine size and that glial processes are more stable around larger spines.

\section{Discussion}

Structural plasticity of the CNS is widely believed to be important for brain function (Segal, 2005; Haber and Murai, 2006). Although most studies have focused on morphological plasticity of dendritic spines, little is known about the properties of astrocytic processes that modulate synaptic function. Historically, glia have been depicted as static elements of the nervous system that simply provide metabolic and structural support for neurons (Volterra and Meldolesi, 2005). Here we provide evidence that astrocytes show remarkable morphological plasticity over minutes and dis- play intricate process extension and retraction events that likely use the actin cytoskeleton. Using simultaneous manipulation of neurons and glia in hippocampal slices, we characterized the complex morphological interplay between astrocytes and postsynaptic dendritic spines. Astrocytic movements, although not necessarily synchronized with spine motility, show cooperative changes with spines. Furthermore, astrocytes can continuously redefine their juxtaposition to the postsynaptic surface, and the extent of this reorganization appears to be related to spine size. Our results suggest that concerted structural changes of both neurons and glia contribute to synaptic plasticity in the hippocampus.

Spine movements likely change the association of synapses with components in the surrounding extracellular space, including glial cell processes (Dunaevsky et al., 2001). Serial electron microscopy indicates that $\sim 57 \%$ of hippocampal synapses are 

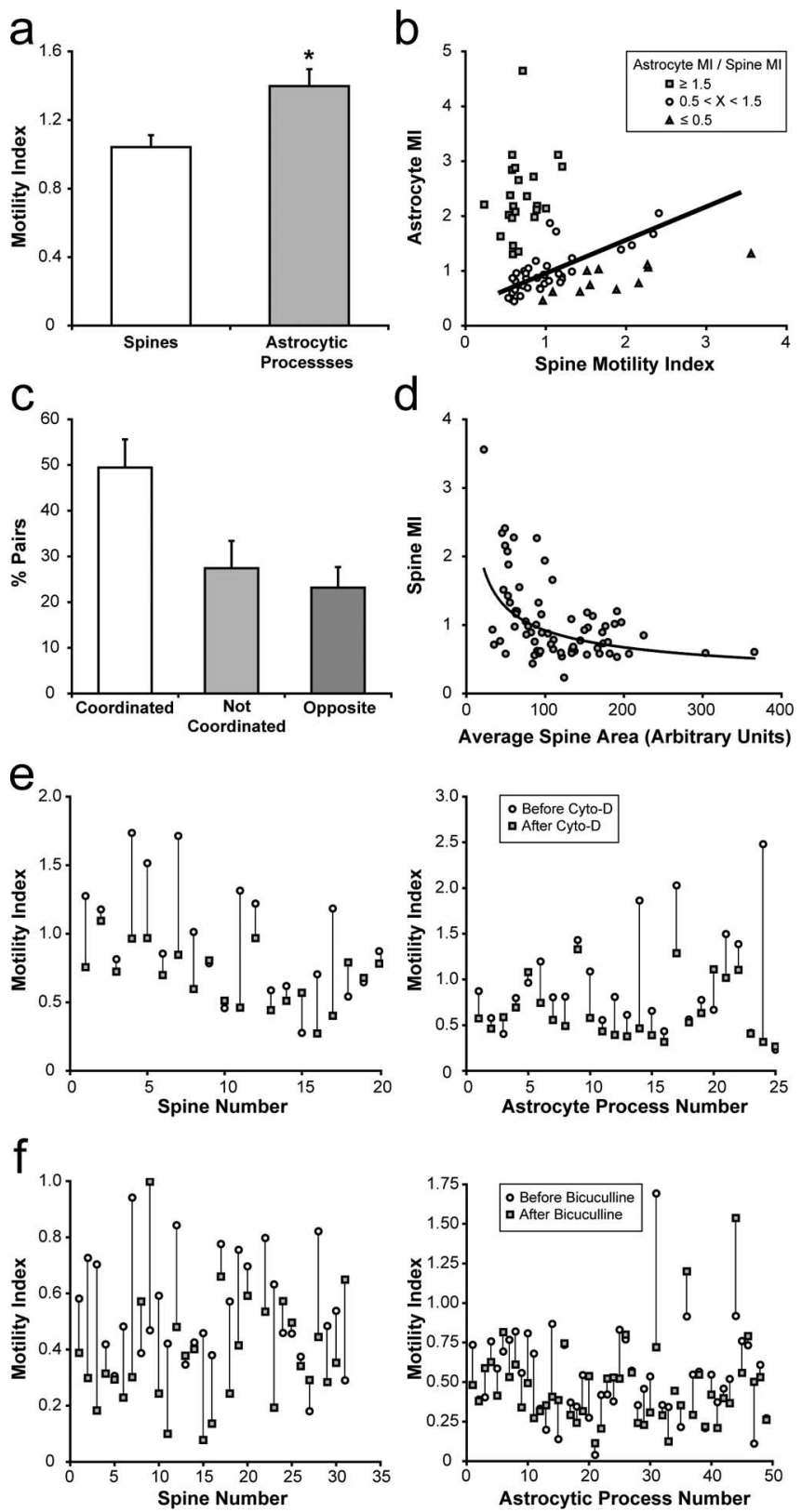

Figure 7. Properties of astrocyte-spine interactions. $\boldsymbol{a}$, Astrocytic processes have significantly higher motility indices (MI) than spines ( $n=71$ spine-astrocyte pairs from 7 experiments; $p<0.007$, paired Student's $t$ test; error bars represent SEM). $\boldsymbol{b}$, Plot of astrocyte versus spine motility reveals three populations of interactions: astrocytes with similar $(51 \%$, astrocyte $\mathrm{MI} /$ spine $\mathrm{Ml}$ is $>0.5$ but $<1.5$; circles, line represents best fit), greater (32\%, astrocyte $\mathrm{MI} /$ spine Ml is $>1.5$; squares), or less ( $17 \%$, astrocyte $\mathrm{Ml} /$ spine $\mathrm{Ml}$ is $<0.5$; triangles) motility than dendritic spines. c, Approximately $50 \%$ of astrocytic process-spine pairs show coordinated changes in size over time, $27 \%$ are not coordinated, and $23 \%$ show opposite changes $(10 \%$ cutoff for increases or decreases in size; see Materials and Methods; error bars represent SEM). $\boldsymbol{d}$, Plot of spine motility index versus average spine area. Spines with greater area show less motility. Curve represents a power trend. $\boldsymbol{e}$, Cytochalasin-D treatment $(5 \mu \mathrm{M})$ reduces both spine and astrocyte motility. Motility indexes calculated before (circles) and after (squares) the start of cytochalasin-D (Cyto-D) treatment for individual spines and astrocytic processes (spines, $p<0.003$, paired Student's $t$ test, $n=20$ spines; astrocytic processes, $p<0.006$, paired Student's $t$ test, $n=25$ astrocytic processes). $\boldsymbol{f}$, Perfusion of bicuculline ( $20 \mu \mathrm{M}$ ) significantly reduces the motility of dendritic spines but not astrocytic processes. Motility indexes calculated before (circles) and after (squares) the start of bicuculline application for spines and astrocytic process (spines, $p=0.002$, paired Student's $t$ test, $n=31$ spines; astrocytic processes, $p>$ 0.05 , paired Student's $t$ test, $n=49$ astrocytic processes).
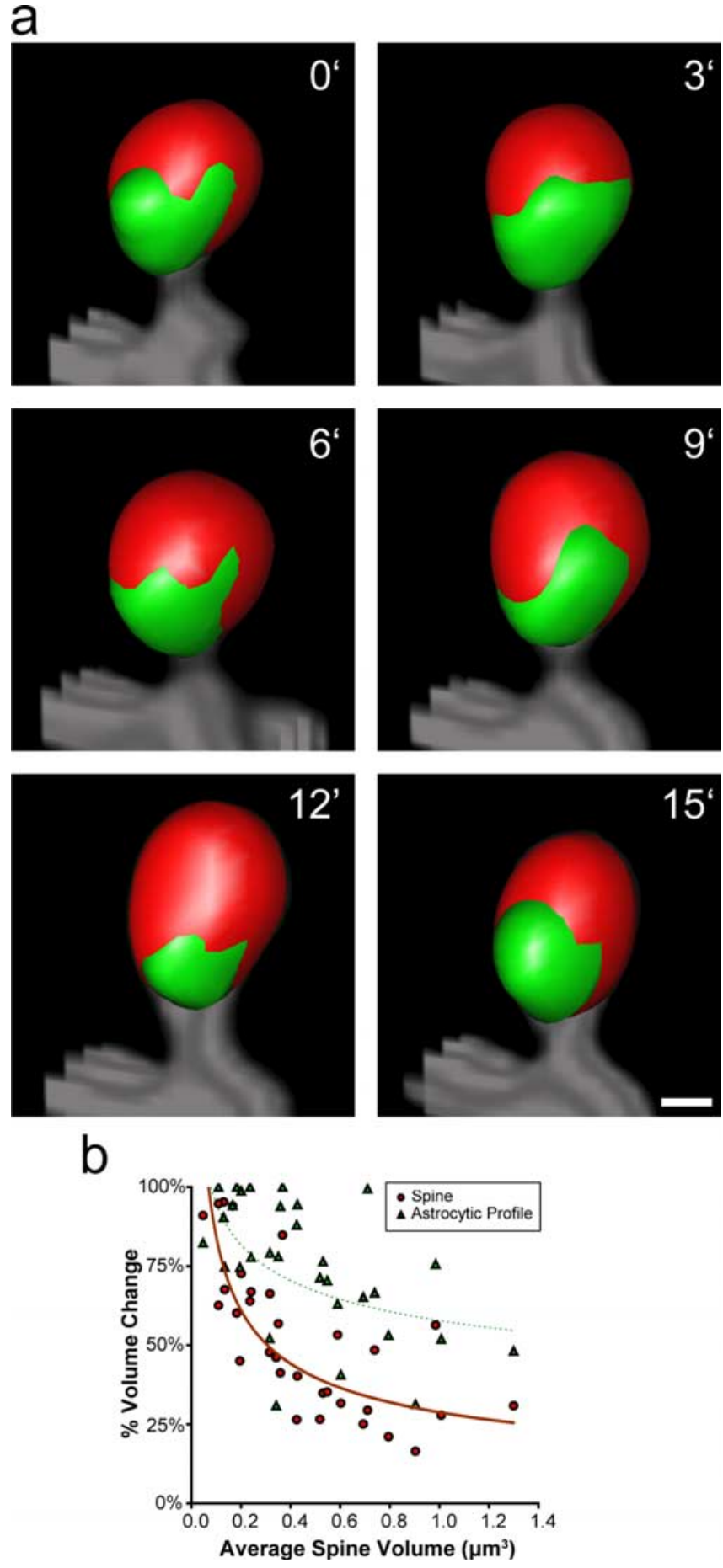

Figure 8. Stability of astrocyte-spine interactions. $\boldsymbol{a}$, Example of an interaction between a stable spine and its associated astrocytic process during a 15 min imaging period. $\boldsymbol{b}$, Plot of percentage spine volume change versus average spine volume showing that larger spines have smaller changes in volume over time and more stable contacts with astrocytic processes $(n=$ 32 pairs from 3 experiments). Curves represent power trends. Scale bar, $0.5 \mu \mathrm{m}$.

juxtaposed with astrocytic processes at a given time (Ventura and Harris, 1999). This is consistent with our results demonstrating that many spine-astrocyte interactions are transient and that only a subset of spines may be in direct contact with an astrocyte at a particular moment. Remarkably, processes of astrocytes are, on average, more motile than their dendritic spine counterparts. Thus, astrocytic motility may have a greater impact on governing the extent of glial coverage over synapses in the hippocampus. 
The proximity of astrocytes to dendritic spines in the hippocampus is known to be different from neuron-glial interactions in other systems, such as the cerebellum in which Bergmann glial extensions can fully encapsulate parallel fiber/Purkinje neuron synapses (Grosche et al., 2002). The arborization of Bergmann glial processes is organized into discrete microdomains that respond independently to synaptic activity (Grosche et al., 1999). The overall extent of glial proximity at spines may reflect the basal physiological level of neuron-glial interplay that takes place at different synapse subtypes. However, like in the hippocampus, Bergmann glia processes appear to have a preference for localizing near postsynaptic dendritic spines (Lehre and $\mathrm{Ru}$ sakov, 2002). This may indicate a common function for glial appendages in modulating postsynaptic function. It would be interesting to determine whether Bergmann glia have more stable contacts with Purkinje neuron spines than astrocytic processes with CA1 pyramidal cell spines in the hippocampus.

What is the physiological importance of dynamic neuronglial interactions in the brain? Astrocyte motility may regulate the positioning of key molecules (i.e., glutamate transporters) that actively control the properties of the synaptic microenvironment (Huang and Bergles, 2004). This is in addition to the ability of astrocytes to partition the extracellular space and influence the diffusion of neuroactive substances (Sykova, 2004). This could control the degree of glutamate spillover onto neighboring synapses and modify heterosynaptic plasticity (Kullmann and Asztely, 1998; Panatier and Oliet, 2006). The positioning of astrocytic processes may also serve to localize glial machinery for vesicular-mediated release of glutamate, ATP, and D-serine in response to synaptic activity (Bezzi et al., 1998, 2004; Kreft et al., 2004; Zhang et al., 2004; Mothet et al., 2005). ATP released from astrocytes and its subsequent breakdown to adenosine suppresses synaptic transmission through adenosine receptors and possibly expands the range of synaptic plasticity (Pascual et al., 2005). D-Serine, which binds to the high-affinity glycine site of NMDA receptor subunits, can directly regulate channel function and modulate LTP (Yang et al., 2003; Panatier et al., 2006). Thus, structural modifications to astrocytes may have widespread affects on the physiological properties of synapses.

Simultaneous modifications of neuronal and glial cell morphology are known to take place in the brain and have been linked to behavior. For instance, naturally occurring changes in dendrites and astrocytes occur during the estrus cycle. Dendritic spine density is increased on pyramidal cells (Woolley et al., 1990), whereas astrocyte volume becomes lower in area CA1 of the hippocampus during proestrus, a time when estrogen levels peak during the reproductive cycle (Klintsova et al., 1995). Structural changes in spines and astrocytes have also been reported to take place after LTP induction (Wenzel et al., 1991; Segal, 2005), experimental models of epilepsy (Hawrylak et al., 1993; McKinney, 2005; Zha et al., 2005), and when rodents are reared in an enriched environment (Berman et al., 1996; Jones and Greenough, 1996; Soffie et al., 1999). Astrocytes also modulate synaptic efficacy of magnocellular neurons of the supraoptic nucleus of the hypothalamus by extending and retracting their processes. This remodeling occurs during lactation, pregnancy, and episodes of dehydration and coincides with differential neuronal firing patterns (Panatier and Oliet, 2006). However, the time course (minutes, hours, or days) of these events and the properties of the glial changes involved remain to be clarified.

Our results demonstrate that morphological changes in astrocytes can be rapid, occurring within minutes in hippocampal slice culture. This may be important for physiological changes that are induced over short time periods such as LTP. However, it should be mentioned that organotypic slice cultures cannot not fully mimic the properties of the intact brain. Although neuronal remodeling is remarkably similar in organotypic cultures and in vivo (Dunaevsky et al., 1999; Grutzendler et al., 2002; Trachtenberg et al., 2002; Oray et al., 2006), associational/commissural and afferent connections of the hippocampus with other brain regions are lost in organotypic preparations. This could alter the degree of activity of astrocytes and influence their motility. Although in vivo imaging has its own limitations with regard to imaging glia (i.e., potential acute damage to the brain, the need of anesthesia), it will likely enable a full appreciation of the extent of astrocytic remodeling in the brain.

Interestingly, accumulating evidence indicates that glutamate controls the structural features of both neurons and glial cells. For example, glutamate stabilizes spines when spontaneously released from presynaptic terminals (McKinney et al., 1999) and causes the formation of small protrusions from the spine head when focally applied (Richards et al., 2005). Glutamate signaling through AMPA receptors is known to stabilize the actin cytoskeleton and spine motility (Fischer et al., 2000). Astrocytes also display a structural response to glutamate by increasing the number of surface filopodia within minutes of its application to dissociated glial cultures (Cornell-Bell et al., 1990). Furthermore, overlaying neurons on monolayers of cultured astrocytes induces astrocytic filopodia formation at sites of membrane contact, an effect that is potentially caused by the local release of neurotransmitter (Cornell-Bell et al., 1990). In the cerebellum, activation of AMPA receptors on Bergmann glia is also required for the maintenance of neuron-glial interactions (Iino et al., 2001). Thus, changes in glutamatergic transmission, as occurs during shortand long-term synaptic plasticity, may lead to rapid and concerted structural modifications of neurons and glial cells.

Perhaps the level of synaptic activity determines the extent of astrocytic association with the synapse. Recent data indicate that increases and decreases in synaptic efficacy accompany spine enlargement and retraction, respectively. This correlates with our data suggesting that spine and astrocytic growth and retraction are coordinated in $\sim 50 \%$ of the cases. Furthermore, astrocytic processes appear to be more stable around spines with larger volume. This is consistent with the finding that astrocytic processes are prevalent around the majority of large, perforated synapses that have a discontinuous postsynaptic density (Ventura and Harris, 1999). Astrocytes may be attracted to larger synapses with potentially greater neurotransmitter release probability (Harris and Sultan, 1995). However, globally reducing inhibitory transmission with bicuculline decreases spine dynamics, although not significantly changing overall astrocytic motility. Thus, astrocytes appear to retain their ability to actively remodel under conditions in which spine motility is generally suppressed. Intercellular signaling through molecules such as ephrins and Eph receptors could help coordinate the reorganization of neuron-glial interactions, ultimately regulating the stability of the synapse (Murai et al., 2003; Haber and Murai, 2006). Future studies will use this system to determine how activity may change the astrocytic-synapse relationship and elucidate the molecular machinery involved.

\section{References}

Benediktsson AM, Schachtele SJ, Green SH, Dailey ME (2005) Ballistic labeling and dynamic imaging of astrocytes in organotypic hippocampal slice cultures. J Neurosci Methods 141:41-53.

Berman RF, Hannigan JH, Sperry MA, Zajac CS (1996) Prenatal alcohol 
exposure and the effects of environmental enrichment on hippocampal dendritic spine density. Alcohol 13:209-216.

Bezzi P, Carmignoto G, Pasti L, Vesce S, Rossi D, Rizzini BL, Pozzan T, Volterra A (1998) Prostaglandins stimulate calcium-dependent glutamate release in astrocytes. Nature 391:281-285.

Bezzi P, Gundersen V, Galbete JL, Seifert G, Steinhauser C, Pilati E, Volterra A (2004) Astrocytes contain a vesicular compartment that is competent for regulated exocytosis of glutamate. Nat Neurosci 7:613-620.

Bishop DL, Misgeld T, Walsh MK, Gan WB, Lichtman JW (2004) Axon branch removal at developing synapses by axosome shedding. Neuron 44:651-661.

Bushong EA, Martone ME, Jones YZ, Ellisman MH (2002) Protoplasmic astrocytes in CA1 stratum radiatum occupy separate anatomical domains. J Neurosci 22:183-192.

Bushong EA, Martone ME, Ellisman MH (2004) Maturation of astrocyte morphology and the establishment of astrocyte domains during postnatal hippocampal development. Int J Dev Neurosci 22:73-86.

Castonguay A, Levesque S, Robitaille R (2001) Glial cells as active partners in synaptic functions. Prog Brain Res 132:227-240.

Christopherson KS, Ullian EM, Stokes CC, Mullowney CE, Hell JW, Agah A, Lawler J, Mosher DF, Bornstein P, Barres BA (2005) Thrombospondins are astrocyte-secreted proteins that promote CNS synaptogenesis. Cell 120:421-433.

Cornell-Bell AH, Thomas PG, Smith SJ (1990) The excitatory neurotransmitter glutamate causes filopodia formation in cultured hippocampal astrocytes. Glia 3:322-334.

Dailey ME, Smith SJ (1996) The dynamics of dendritic structure in developing hippocampal slices. J Neurosci 16:2983-2994.

DiCiommo DP, Bremner R (1998) Rapid, high level protein production using DNA-based Semliki Forest virus vectors. J Biol Chem 273:18060-18066.

Dunaevsky A, Tashiro A, Majewska A, Mason C, Yuste R (1999) Developmental regulation of spine motility in the mammalian central nervous system. Proc Natl Acad Sci USA 96:13438-13443.

Dunaevsky A, Blazeski R, Yuste R, Mason C (2001) Spine motility with synaptic contact. Nat Neurosci 4:685-686.

Ehrengruber MU, Lundstrom K, Schweitzer C, Heuss C, Schlesinger S, Gahwiler BH (1999) Recombinant Semliki Forest virus and Sindbis virus efficiently infect neurons in hippocampal slice cultures. Proc Natl Acad Sci USA 96:7041-7046.

Ehrengruber MU, Renggli M, Raineteau O, Hennou S, Vaha-Koskela MJ, Hinkkanen AE, Lundstrom K (2003) Semliki Forest virus A7(74) transduces hippocampal neurons and glial cells in a temperature-dependent dual manner. J Neurovirol 9:16-28.

Fischer M, Kaech S, Knutti D, Matus A (1998) Rapid actin-based plasticity in dendritic spines. Neuron 20:847-854.

Fischer M, Kaech S, Wagner U, Brinkhaus H, Matus A (2000) Glutamate receptors regulate actin-based plasticity in dendritic spines. Nat Neurosci 3:887-894.

Gan WB, Grutzendler J, Wong WT, Wong RO, Lichtman JW (2000) Multicolor "DiOlistic" labeling of the nervous system using lipophilic dye combinations. Neuron 27:219-225.

Grosche J, Matyash V, Moller T, Verkhratsky A, Reichenbach A, Kettenmann H (1999) Microdomains for neuron-glia interaction: parallel fiber signaling to Bergmann glial cells. Nat Neurosci 2:139-143.

Grosche J, Kettenmann H, Reichenbach A (2002) Bergmann glial cells form distinct morphological structures to interact with cerebellar neurons. J Neurosci Res 68:138-149.

Grutzendler J, Kasthuri N, Gan WB (2002) Long-term dendritic spine stability in the adult cortex. Nature 420:812-816.

Haber M, Murai KK (2006) Reshaping neuron-glial communication at hippocampal synapses. Neuron Glia Biol 2:59-66.

Harris KM, Sultan P (1995) Variation in the number, location and size of synaptic vesicles provides an anatomical basis for the nonuniform probability of release at hippocampal CA1 synapses. Neuropharmacology 34:1387-1395.

Hawrylak N, Chang FL, Greenough WT (1993) Astrocytic and synaptic response to kindling in hippocampal subfield CA1. II. Synaptogenesis and astrocytic process increases to in vivo kindling. Brain Res 603:309-316.

Haydon PG (2001) GLIA: listening and talking to the synapse. Nat Rev Neurosci 2:185-193.

Hirrlinger J, Hulsmann S, Kirchhoff F (2004) Astroglial processes show spontaneous motility at active synaptic terminals in situ. Eur J Neurosci 20:2235-2239.

Holtmaat AJ, Trachtenberg JT, Wilbrecht L, Shepherd GM, Zhang X, Knott GW, Svoboda K (2005) Transient and persistent dendritic spines in the neocortex in vivo. Neuron 45:279-291.

Huang YH, Bergles DE (2004) Glutamate transporters bring competition to the synapse. Curr Opin Neurobiol 14:346-352.

Iino M, Goto K, Kakegawa W, Okado H, Sudo M, Ishiuchi S, Miwa A, Takayasu Y, Saito I, Tsuzuki K, Ozawa S (2001) Glia-synapse interaction through $\mathrm{Ca}^{2+}$-permeable AMPA receptors in Bergmann glia. Science 292:926-929.

Jones TA, Greenough WT (1996) Ultrastructural evidence for increased contact between astrocytes and synapses in rats reared in a complex environment. Neurobiol Learn Mem 65:48-56.

Kaufmann WE, Moser HW (2000) Dendritic anomalies in disorders associated with mental retardation. Cereb Cortex 10:981-991.

Kim CH, Lisman JE (1999) A role of actin filament in synaptic transmission and long-term potentiation. J Neurosci 19:4314-4324.

Klintsova A, Levy WB, Desmond NL (1995) Astrocytic volume fluctuates in the hippocampal CA1 region across the estrous cycle. Brain Res 690:269-274.

Korkotian E, Segal M (1998) Fast confocal imaging of calcium released from stores in dendritic spines. Eur J Neurosci 10:2076-2084.

Korkotian E, Segal M (2001) Spike-associated fast contraction of dendritic spines in cultured hippocampal neurons. Neuron 30:751-758.

Kreft M, Stenovec M, Rupnik M, Grilc S, Krzan M, Potokar M, Pangrsic T, Haydon PG, Zorec R (2004) Properties of $\mathrm{Ca}^{2+}$-dependent exocytosis in cultured astrocytes. Glia 46:437-445.

Kullmann DM, Asztely F (1998) Extrasynaptic glutamate spillover in the hippocampus: evidence and implications. Trends Neurosci 21:8-14.

Lehre KP, Rusakov DA (2002) Asymmetry of glia near central synapses favors presynaptically directed glutamate escape. Biophys J 83:125-134.

Lundstrom K, Abenavoli A, Malgaroli A, Ehrengruber MU (2003) Novel Semliki Forest virus vectors with reduced cytotoxicity and temperature sensitivity for long-term enhancement of transgene expression. Mol Ther 7:202-209.

Matsuzaki M, Honkura N, Ellis-Davies GC, Kasai H (2004) Structural basis of long-term potentiation in single dendritic spines. Nature 429:761-766.

Matus A (2000) Actin-based plasticity in dendritic spines. Science 290:754-758.

McKinney RA (2005) Physiological roles of spine motility: development, plasticity and disorders. Biochem Soc Trans 33:1299-1302.

McKinney RA, Capogna M, Durr R, Gahwiler BH, Thompson SM (1999) Miniature synaptic events maintain dendritic spines via AMPA receptor activation. Nat Neurosci 2:44-49.

Mothet JP, Pollegioni L, Ouanounou G, Martineau M, Fossier P, Baux G (2005) Glutamate receptor activation triggers a calcium-dependent and SNARE protein-dependent release of the gliotransmitter D-serine. Proc Natl Acad Sci USA 102:5606-5611.

Murai KK, Nguyen LN, Irie F, Yamaguchi Y, Pasquale EB (2003) Control of hippocampal dendritic spine morphology through ephrin-A3/EphA4 signaling. Nat Neurosci 6:153-160.

Nagerl UV, Eberhorn N, Cambridge SB, Bonhoeffer T (2004) Bidirectional activity-dependent morphological plasticity in hippocampal neurons. Neuron 44:759-767.

Niell CM, Meyer MP, Smith SJ (2004) In vivo imaging of synapse formation on a growing dendritic arbor. Nat Neurosci 7:254-260.

Ogata K, Kosaka T (2002) Structural and quantitative analysis of astrocytes in the mouse hippocampus. Neuroscience 113:221-233.

Oray S, Majewska A, Sur M (2006) Effects of synaptic activity on dendritic spine motility of developing cortical layer v pyramidal neurons. Cereb Cortex 16:730-741.

Panatier A, Oliet SHR (2006) Neuron-glia interactions in the hypothalamus. Neuron Glia Biol 2:51-58.

Panatier A, Theodosis DT, Mothet JP, Touquet B, Pollegioni L, Poulain DA, Oliet SH (2006) Glia-derived D-serine controls NMDA receptor activity and synaptic memory. Cell 125:775-784.

Pascual O, Casper KB, Kubera C, Zhang J, Revilla-Sanchez R, Sul JY, Takano H, Moss SJ, McCarthy K, Haydon PG (2005) Astrocytic purinergic signaling coordinates synaptic networks. Science 310:113-116.

Richards DA, De Paola V, Caroni P, Gahwiler BH, McKinney RA (2004) AMPA-receptor activation regulates the diffusion of a membrane marker 
in parallel with dendritic spine motility in the mouse hippocampus. J Physiol (Lond) 558:503-512.

Richards DA, Mateos JM, Hugel S, de Paola V, Caroni P, Gahwiler BH, McKinney RA (2005) Glutamate induces the rapid formation of spine head protrusions in hippocampal slice cultures. Proc Natl Acad Sci USA 102:6166-6171.

Segal M (2005) Dendritic spines and long-term plasticity. Nat Rev Neurosci 6:277-284.

Soffie M, Hahn K, Terao E, Eclancher F (1999) Behavioural and glial changes in old rats following environmental enrichment. Behav Brain Res 101:37-49.

Stoppini L, Buchs PA, Muller D (1991) A simple method for organotypic cultures of nervous tissue. J Neurosci Methods 37:173-182.

Sykova E (2004) Extrasynaptic volume transmission and diffusion parameters of the extracellular space. Neuroscience 129:861-876.

Trachtenberg JT, Chen BE, Knott GW, Feng G, Sanes JR, Welker E, Svoboda $\mathrm{K}$ (2002) Long-term in vivo imaging of experience-dependent synaptic plasticity in adult cortex. Nature 420:788-794.

Ullian EM, Sapperstein SK, Christopherson KS, Barres BA (2001) Control of synapse number by glia. Science 291:657-661.

Ventura R, Harris KM (1999) Three-dimensional relationships between hippocampal synapses and astrocytes. J Neurosci 19:6897-6906.

Volfovsky N, Parnas H, Segal M, Korkotian E (1999) Geometry of dendritic spines affects calcium dynamics in hippocampal neurons: theory and experiments. J Neurophysiol 82:450-462.

Volterra A, Meldolesi J (2005) Astrocytes, from brain glue to communication elements: the revolution continues. Nat Rev Neurosci 6:626-640.
Walsh MK, Lichtman JW (2003) In vivo time-lapse imaging of synaptic takeover associated with naturally occurring synapse elimination. Neuron 37:67-73.

Wenzel J, Lammert G, Meyer U, Krug M (1991) The influence of long-term potentiation on the spatial relationship between astrocyte processes and potentiated synapses in the dentate gyrus neuropil of rat brain. Brain Res 560:122-131.

Woolley CS, Gould E, Frankfurt M, McEwen BS (1990) Naturally occurring fluctuation in dendritic spine density on adult hippocampal pyramidal neurons. J Neurosci 10:4035-4039.

Yang Y, Ge W, Chen Y, Zhang Z, Shen W, Wu C, Poo M, Duan S (2003) Contribution of astrocytes to hippocampal long-term potentiation through release of D-serine. Proc Natl Acad Sci USA 100:15194-15199.

Zha X, Green SH, Dailey ME (2005) Regulation of hippocampal synapse remodeling by epileptiform activity. Mol Cell Neurosci 29:494-506.

Zhang Q, Pangrsic T, Kreft M, Krzan M, Li N, Sul JY, Halassa M, Van Bockstaele E, Zorec R, Haydon PG (2004) Fusion-related release of glutamate from astrocytes. J Biol Chem 279:12724-12733.

Zhou J, Sutherland ML (2004) Glutamate transporter cluster formation in astrocytic processes regulates glutamate uptake activity. J Neurosci 24:6301-6306.

Zhou Q, Homma KJ, Poo MM (2004) Shrinkage of dendritic spines associated with long-term depression of hippocampal synapses. Neuron 44:749-757.

Zuo Y, Lin A, Chang P, Gan WB (2005) Development of long-term dendritic spine stability in diverse regions of cerebral cortex. Neuron 46:181189. 